\title{
INPUT-OUTPUT RELATIONSHIPS OF CENTRAL NEURAL CIRCUITS INVOLVED IN RESPIRATION IN CATS
}

\author{
By FREDERIC L. ELDRIDGE, PRITAM GILL-KUMAR* \\ AND DAVID E. MILLHORN \\ From the Departments of Medicine and Physiology, University of North Carolina, \\ Chapel Hill, North Carolina 27514, U.S.A.
}

(Received 25 March 1980)

\section{SUMMARY}

1. Inspiratory output responses, measured as integrated phrenic activity, to hypercapnia, to unilateral and bilateral carotid sinus nerve stimulation and to combinations of these stimuli were determined in paralysed, vagotomized and glomectomized cats whose end-tidal $P_{\mathrm{CO}_{2}}$ was kept constant by means of a servocontrolled ventilator. In addition, the effect on these responses of the mechanism that causes the respiratory after-discharge was determined.

2. Above the threshold for rhythmic activity, the inspiratory response to hypercapnic stimulation of the central chemoreceptor was curvilinear, showing progressively smaller increments of output for equal increments of $P_{\mathrm{CO}_{2}}$ as the latter became higher.

3. The combining of stimuli from right and left carotid sinus nerves failed to show an algebraically additive effect; the response was approximately $70 \%$ of that predicted from a summing of the separate stimuli given alone.

4. The response to a constant carotid sinus nerve test stimulus was progressively decreased in magnitude as the pre-stimulus level of respiratory activity was increased by conditioning stimulation of the central chemoreceptors by hypercapnia, by stimulation of the opposite carotid sinus nerve or by the mechanism that generates an after-discharge.

5. From a descriptive standpoint, our findings show that there is a negative or hypoadditive interaction between the peripheral and central inputs at the level of the central respiratory controller. However, we present evidence that, rather than being a specific interaction between peripheral and central inputs, the response is due to the properties of a neural component of the central pathway. This component is common to both inputs and develops progressive saturation of its neural elements as its activity increases.

6. In addition, the neural mechanism which generates a respiratory after-discharge appears to saturate completely at a lower level of inspiratory activity than that at which the common pathway develops complete saturation. This finding supports the idea that this mechanism represents an independent input to the respiratory controller.

* Present address: Department of Physiology and Biophysics, College of Medicine, Howard University, Washington, D.C. 20059, U.S.A. 
7. Because the described a-linear response characteristics of the central respiratory controller are due to its inherent neuronal properties rather than to specific interactions between inputs, we suggest that studies of such 'interactions' must be interpreted with this consideration in mind.

\section{INTRODUCTION}

Afferent input from either peripheral or central chemoreceptors causes respiration to increase. Although there are a number of studies addressing the problem, the nature of the interaction between the two inputs when given together remains a matter of disagreement. Indeed, it has been claimed that the interaction is a positive one, i.e., the presence of one stimulus augments the other in a synergistic or multiplicative fashion that it is simply additive, or that it is negative or hypoadditive.

A variety of factors probably account for these differences. There is little doubt that a positive or synergistic interaction between hypoxia and hypercapnia occurs in the carotid body (Eyzaguirre \& Lewin, 1961) and can affect the results in intact animals. If the vagus nerves remain intact, receptors sensitive to airway $\mathrm{CO}_{2}$ (Mustafa \& Purves, 1972) can be partly responsible. The use of systemic hypoxia can lead to complicating effects, such as changing cerebral circulation, medullary neuronal depression (Cherniack, Edelman \& Lahiri, 1970/1971), to an increased concentration of lactic acid in the brain and c.s.f. (Sorenson, 1970), and activation of diencephalic facilitatory mechanisms (Tenney \& Ou, 1977).

Because of these problems it has been difficult to determine what interactions, if any, occur solely at the level of the neural respiratory controller itself. In previous studies we showed that the respiratory response to a constant carotid sinus nerve test stimulus decreased progressively as $P_{\mathrm{CO}_{2}}$ rose (Eldridge, 1974 ; see Fig. 4), and that a similar interaction occurred between a constant carotid sinus nerve test stimulus and the mechanism which produces the respiratory after-discharge (Eldridge \& Gill-Kumar, 1980a). We suggested that this interaction could be due to progressive saturation of some neural component of the central processor but could not rule out occlusion of neural inputs at their point of convergence. Based on similar responses to hypoxic and hypercapnic inputs, and to two separate carotid sinus nerve inputs, Kiwull, Kiwull-Schöne \& Klatt (1976) concluded that the hypoadditive interaction was due to occlusion.

The present study was designed to examine this question further. In order to avoid central and peripheral feedback, the experiments were performed in paralysed animals whose vagi and carotid sinus nerves were sectioned and whose end-tidal $\boldsymbol{P}_{\mathrm{CO}_{2}}$ was kept constant during a given experiment by means of a servo-controlled ventilator. Instead of using hypoxia to stimulate the carotid body, we mimicked carotid body afferent input by stimulating the carotid sinus nerve electrically. We believe this preparation avoids the complicating problems of hypoxia on circulation, neural function and suprapontine brain and has allowed us to examine the true neural controller interactions to several peripheral and central respiratory stimulus inputs. 


\section{METHODS}

Studies were performed in seventeen healthy adult cats weighing between $2 \cdot 2$ and $4 \cdot 4 \mathrm{~kg}$. They were anaesthetized with ether and then given chloralose $(40 \mathrm{mg} / \mathrm{kg})$ and urethane $(250 \mathrm{mg} / \mathrm{kg})$ via a foreleg vein. Femoral artery pressure was measured by means of a strain gauge. Temperature was monitored with a rectal thermistor and servo-controlled at $37.5^{\circ} \mathrm{C}$ by means of an electronic circuit and DC heating pad. The trachea was cannulated through a neck incision and continuous sampling of airway $\mathrm{CO}_{2}$ was accomplished by means of a catheter placed in the airway; analysis was made by an infra-red $\mathrm{CO}_{2}$ analyser (Beckman LB-2).

Following these preparations, the animal was placed supine on a table with a rigid head mount. Both vago-sympathetic trunks were cut in the neck. One phrenic nerve root (C5) was also exposed in the neck, cut, desheathed and placed on a bipolar platinum recording electrode. Both carotid sinus nerves (c.s.n.) were exposed and crushed near the carotid bodies; one or both nerves were then placed on separate bipolar platinum stimulating electrodes. The nerves and electrodes were immersed in pools of mineral oil. All three of these electrodes were built into small acrylic 'boats' which could be placed in the tissue wells adjacent to the respective nerves and the nerves laid over them. Because the electrodes had no fixed external attachments (the electrical wires were flexible and moved freely) they moved with the cat. With this technique it was possible to leave the nerves and electrodes untouched even during a long experiment and to maintain a relatively constant electrical coupling between nerve and electrode.

The animal was then ventilated with $100 \% \mathrm{O}_{2}$ using a volume-cycled ventilator and paralysed with gallamine triethiodide, $3 \mathrm{mg} / \mathrm{kg}$ I.v. initially, with additional doses as necessary to prevent muscular activity. In order to prevent significant changes in end-tidal and arterial $P_{\mathrm{CO}_{2}}$ secondary to changes in cardiac output and venous $\mathrm{CO}_{2}$ return to the lungs, the $\mathrm{DC}$ voltage on the ventilator's motor was controlled by the animal's end-tidal $P_{\mathrm{CO}}$, through an electronic circuit. The ventilator's rate was thereby servo-controlled to maintain $P_{\mathrm{ET}}$, co, within \pm 0.5 torr of the set level (Smith, Mercer \& Eldridge, 1978). When hypercapnia was to be induced in an animal, dead spaces of various sizes were introduced into the ventilatory circuit and the set-point of the servo-controller changed to the desired level of $P_{\mathrm{CO}_{2}}$.

Stimulation of the carotid sinus nerves was performed with stimulus trains having a frequency of $25 \mathrm{~Hz}$ and $0.5 \mathrm{msec}$ pulse duration. Voltage was varied to obtain different levels of stimulation needed for the experiments.

\section{Experimental protocols}

Response to hypercapnia (seventeen cats). After determining the $P_{\mathrm{CO}}$ at the threshold of rhythmic inspiratory activity, the end-tidal $P_{\mathrm{CO}_{2}}$ was raised. After $10 \mathrm{~min}$, when the level of phrenic activity had become stable, the values were recorded. Additional step increases of $P_{\mathrm{CO}}$ to as high as 40 torr above threshold were then performed and the response at each step determined in a similar manner.

Response to combined stimuli (nine cats). Initial, or control conditions were recorded after attaining a fixed $P_{\mathrm{ET}, \mathrm{CO}_{z}}$ and stable inspiratory output. Then one of the c.s.n. was stimulated (the test stimulus) either continuously or during alternate breaths over a period of $1 \mathrm{~min}$, and the phrenic response recorded. Following offset of the stimulus, the decaying after-discharge was recorded for another $5 \mathrm{~min}$. Three types of experiments were performed:

(1) The phrenic response to a continuous c.s.n. test stimulus was first determined. Then the opposite c.s.n. was stimulated in the same manner and its response measured. Finally both c.s.n.s were stimulated simultaneously, using the original stimulus variables for each, and the combined response recorded.

(2) $P_{\mathrm{ET}, \mathrm{CO}}$, was set at a level which yielded a phrenic output in the range of the spontaneously breathing cat before paralysis. The response to a test stimulation of one c.s.n. was determined. Then the opposite c.s.n. was stimulated continuously with voltage sufficient to raise the phrenic activity to a stable higher level; this conditioning stimulus was continued during the reactivation of the first, or test, c.s.n. stimulation and throughout the period of the after-discharge which followed the offset of the test stimulus. Finally a still higher conditioning c.s.n. stimulus was given and the test stimulus repeated.

(3) In these experiments increased $\mathrm{CO}_{2}$ was the conditioning stimulus. After recording the response to a test c.s.n. stimulus $\boldsymbol{P}_{\mathrm{ET}, \mathrm{CO}_{2}}$ was raised as above. When stable conditions had been 
achieved, the test c.s.n. stimulus response was again recorded. Finally a still higher level of $P_{\mathrm{ET}, \mathrm{CO}_{2}}$ was induced and the test stimulus repeated.

Data handling. Data obtained included arterial pressure, airway $P_{\mathrm{CO}_{2}}$, phrenic nerve impulses and markers for both right and left c.s.n. stimulation, all of which were recorded on magnetic tape. Breath-by-breath analysis of all runs was performed by a computer. Phrenic activity was half-wave recitified and integrated for each 0.1 sec period by means of an integrating digital voltmeter as previously described (Eldridge, 1975). The inspiratory (tidal) output for each breath was determined from the peak $0 \cdot 1$ sec activity. Neural minute activity was calculated as the product of this tidal value and respiratory frequency.

In order to compare the findings among different cats, the data were normalized by assigning a value of 100 units to the maximum activity found in an individual cat during the series of experiments. This level always occurred during combinations of the highest c.s.n. and $\mathrm{CO}_{2}$ stimulations. All other levels of activity were scaled accordingly.

For presentation of results, all values of the control segment were averaged. During test stimulation, values were averaged for each 10 sec period. During the after-discharge, the first post-stimulus breath was calculated separately; the remainder of the recovery period was divided into segments of $10 \mathrm{sec}$ for the first $0.5 \mathrm{~min}, 15 \mathrm{sec}$ for up to $2 \mathrm{~min}, 20 \mathrm{sec}$ to $3 \mathrm{~min}$, and $30 \mathrm{sec}$ for times over $3 \mathrm{~min}$, and the breaths within each time segment averaged.

\section{RESULTS}

\section{Response to hypercapnia}

All seventeen animals were responsive to the hypercapnic stimulus acting only on the central chemoreceptors. The averaged responses for tidal (peak phrenic) neural activity (Fig. $1 \mathrm{~A}$ ) and minute neural activity (Fig. $1 B$ ) are similar because changes of respiratory frequency were relatively small. There is a clear tendency for the response lines to curve to the right, showing that there are progressively smaller increments of inspiratory activity for equal increments of $P_{\mathrm{CO}_{2}}$ as the latter becomes progressively higher.

\section{Responses to combined stimuli}

In order to facilitate understanding of the measurements made on the data of this study, a typical response to continuous c.s.n. test stimulation is shown in Fig. $2 A$. At the left (control) is the initial phrenic activity, which was set basically by the animal's $P_{\mathrm{CO}_{2}}\left(P_{\mathrm{ET}, \mathrm{CO}_{2}}\right.$ was 29 torr in this run) but could be raised by adding a conditioning stimulus from the opposite c.s.n. or by increasing the $P_{\mathrm{CO}_{2}}$ as a conditioning stimulus. The increment of activity over the initial level during the last 20 sec of test stimulation was used to represent the overall effect of a stimulation. At the offset of a stimulus activity remains higher than the initial level. This residual effect has been termed an after-discharge; it is thought to reflect the activity in a neural network which is separate from that of the inspiratory neurones (Eldridge \& Gill-Kumar, 1978). The activity increases slowly during a period of stimulation (Eldridge \& Gill-Kumar, 1979) and decays slowly for some minutes after offset of stimulation (Eldridge, 1974). The increment over the initial level of phrenic activity measured in the first breath after offset of stimulation, when decay would have been minimal, was used to represent the magnitude of the change produced by the after-discharge mechanism (Fig. $2 A$ ).

When the c.s.n. test stimulation was given only during alternate breaths, a dual pattern appeared (Fig. $2 B ; P_{\mathrm{CO}_{2}}=30$ torr) which showed both the rising peak activity and the more slowly rising level of activity due to the after-discharge 
mechanism (Eldridge \& Gill-Kumar, 1979, 1980a). The difference between the peak activity during a stimulus and the after-discharge activity following it represented the increment due solely to the immediate effect of the c.s.n. stimulus independent of the effect of the after-discharge mechanism. In this example, the increments for identical stimuli can be seen to be getting smaller as the after-discharge level rises.

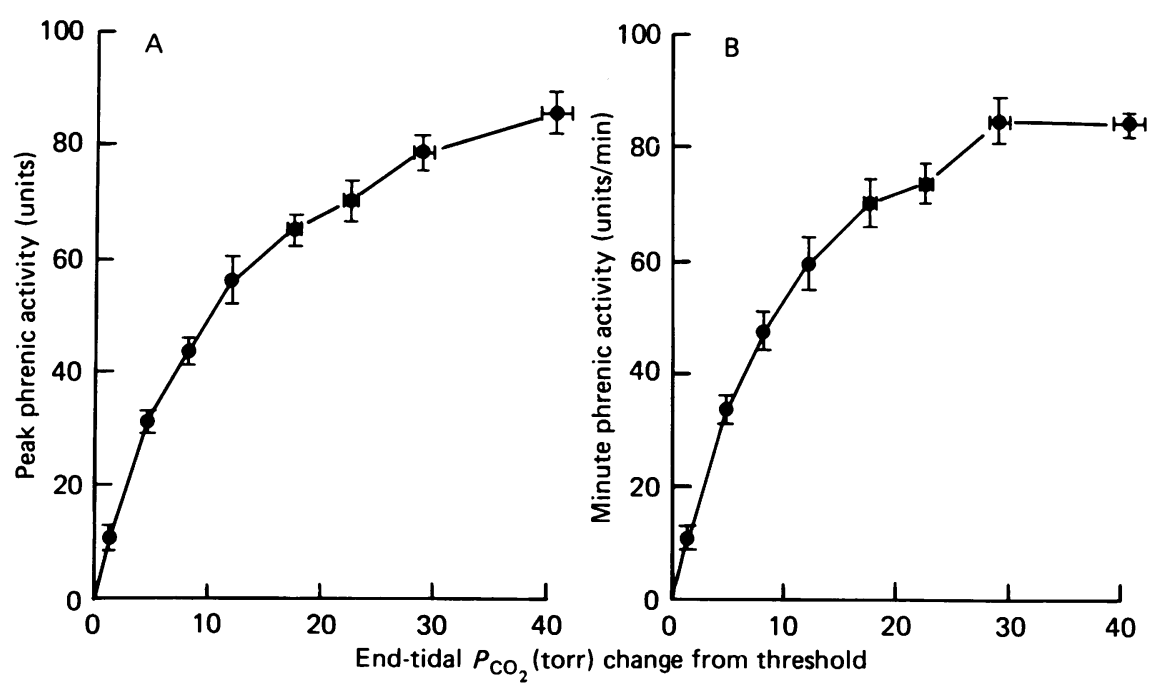

Fig. 1. Relationship between increment over apneic threshold of end-tidal $P_{\mathrm{CO}_{2}}$ and two measures of inspiratory output. $A$, neural tidal (peak phrenic) activity. $B$, neural minute (phrenic) activity.

\section{Unilateral and bilateral carotid sinus nerve stimulation}

The combining of stimuli from right and left c.s.n. did not have an algebraically additive effect (Fig. 3). Fig. $3 A$ shows the response to continuous stimulation of the right c.s.n. and Fig. $3 B$ the response to similar stimulation of the left c.s.n. at the same $P_{\mathrm{ET}, \mathrm{CO}_{2}}$ of 26 torr. When these two stimuli were given simultaneously (Fig. $3 \mathrm{C}$ ) the response increased but was only $70 \%$ of that predicted if the two stimuli were fully additive.

That this effect was not due to complete saturation of neuronal pathways is shown in Fig. 4, where in the same cat at a $P_{\mathrm{ET}, \mathrm{CO}_{2}}$ of 24.5 torr, near apnoeic threshold, the same lack of full addition of the two individual responses was manifest at low, intermediate and high levels of c.s.n. stimulation. The actual combined responses ranged from $\mathbf{7 0}$ to $\mathbf{7 7} \%$ of those predicted if the responses had simply added. Even at the highest stimulus level, the combined response was significantly less than the maximum achievable in this cat with combined c.s.n. and $P_{\mathrm{CO}_{2}}(47$ torr) stimuli. The findings were essentially the same for tidal neural activity (Fig. 4) and minute neural activity, so frequency effects were of little importance. 
Effect of conditioning stimulation by the carotid sinus nerve

In these experiments, the responses to identical c.s.n. test stimuli were determined at different levels of initial (control) respiratory output activity, produced by continuous conditioning stimulation of the opposite c.s.n. The experiments were carried out under conditions of constant $P_{\mathrm{ET}, \mathrm{CO}_{2}}$. A typical example is shown in Fig.

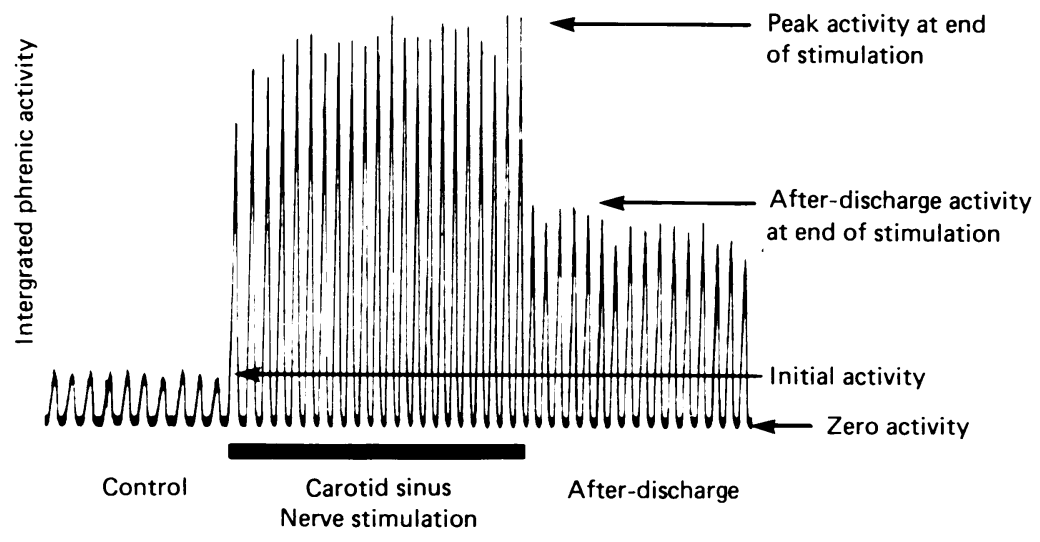

$B$
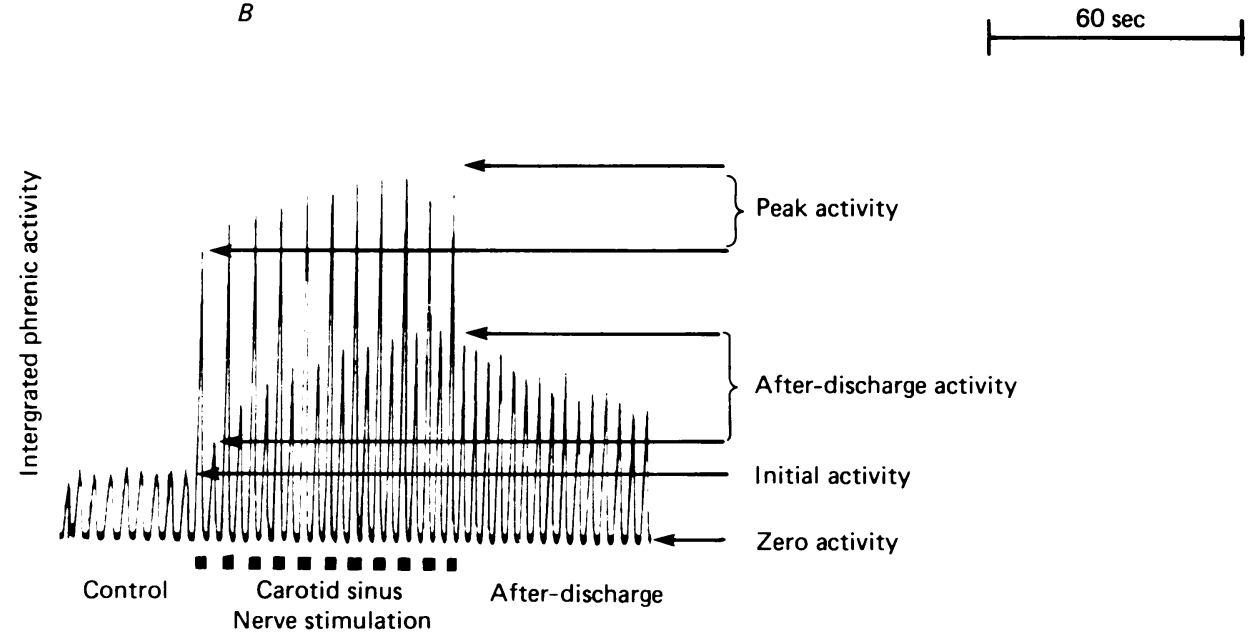

Fig. 2. Measurements of various levels of phrenic activity made in present study. $A$, during continuous carotid sinus nerve stimulation. $B$, during alternate breath carotid sinus nerve stimulation.

5. In Fig. $5 A$ the right c.s.n. (the test stimulus) was given alone; it caused a usual phrenic response and after-discharge. When a moderate conditioning stimulus (left c.s.n.) was given, raising the initial level of activity, the same test stimulus produced smaller increments in both peak activity and after-discharge, i.e., the difference 


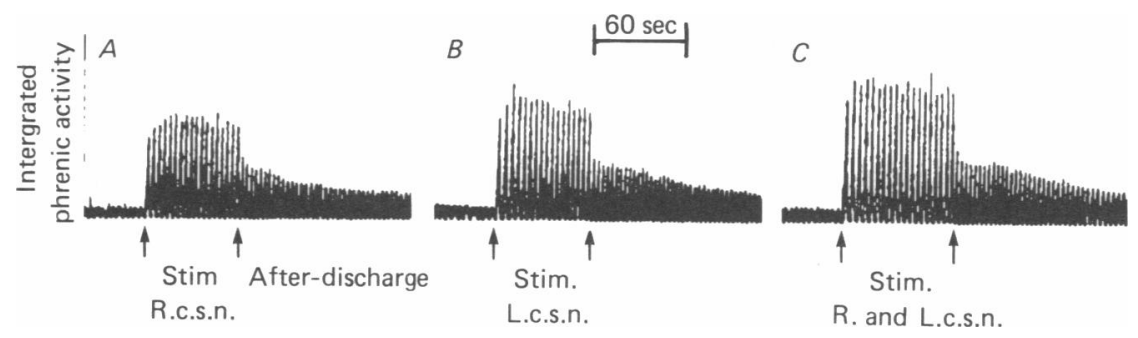

Fig. 3. Responses of phrenic activity to unilateral and bilateral stimulation of carotid sinus nerves (c.s.n.) $A$, right c.s.n. alone. $B$, left c.s.n. alone. $C$, right and left c.s.n. together.

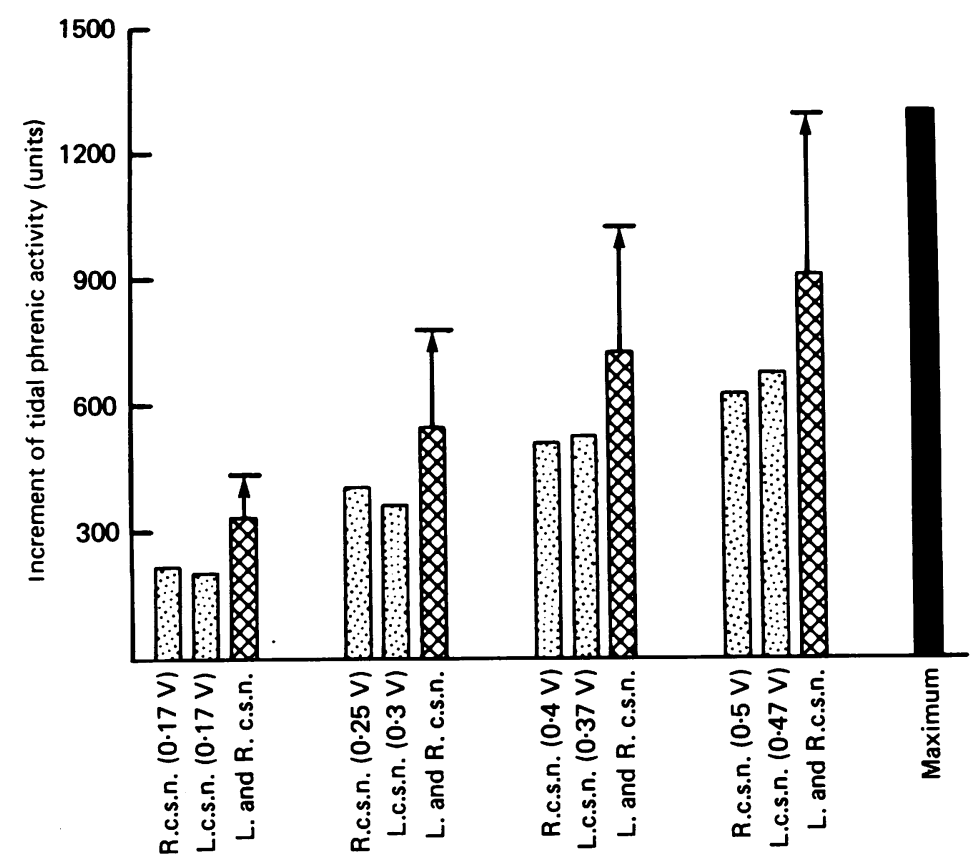

Fig. 4. Increments of neural tidal (peak) phrenic activity during unilateral and bilateral carotid sinus nerve (c.s.n.) stimulations of different intensities. The maximum response (filled column) was the largest obtained in this cat during combined c.s.n. stimulations and hypercapnia.

between the initial level and the first breath of the after-discharge (Fig. $5 B$ ). With a still larger conditioning stimulus and higher initial activity, the test stimulus caused an even smaller peak response and virtual disappearance of the after-discharge process (Fig. $5 \mathrm{C}$ ). When, in the same cat, the right c.s.n. was used as the conditioning stimulus and the left c.s.n. as the test stimulus, the findings were essentially the same.

The combined data for all cats were divided into four approximately equally sized groups depending upon the initial level of activity of each run. The ranges of activities in these groups were 0-20 units, 21-41 units, 42-60 units and 61-80 units. Fig. 6 

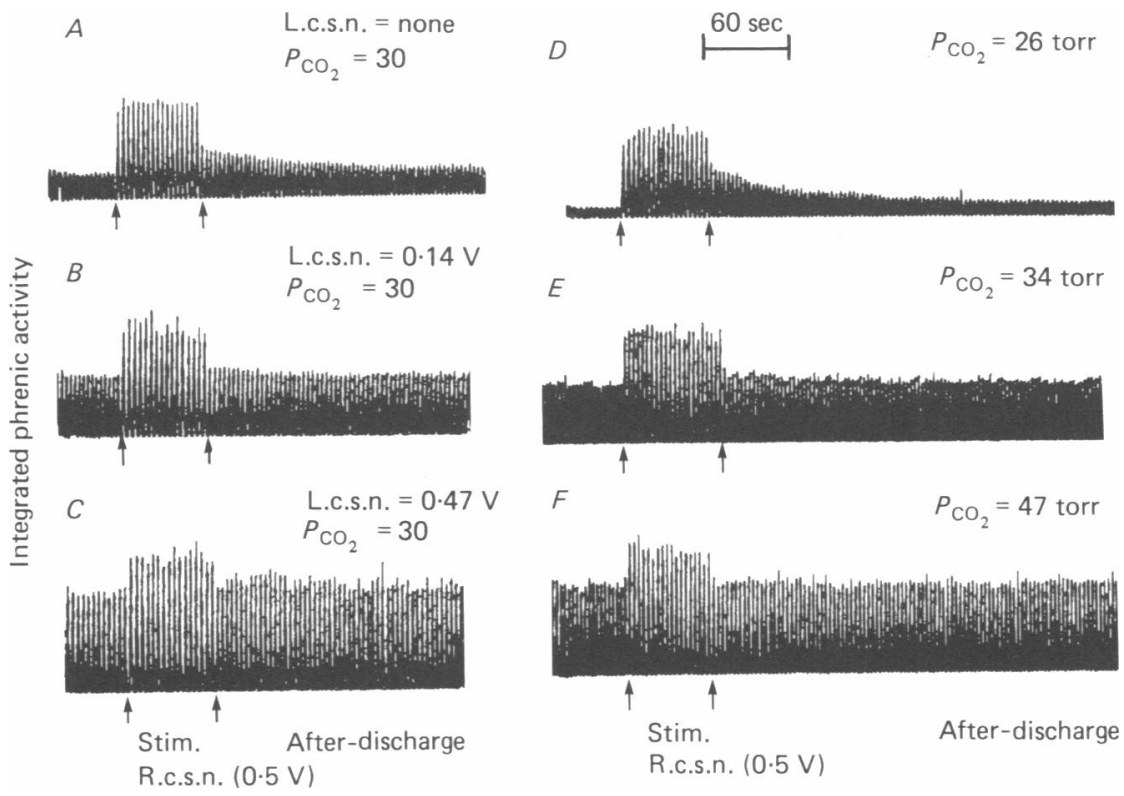

Fig. 5. Responses of phrenic activity to carotid sinus nerve (c.s.n.) test stimulations. $A-C$, under isocapnic conditions with different intensities of conditioning stimulation from opposite c.s.n. $D-F$, with increasing hypercapnic conditioning from the central chemoreceptors.

Both types of conditioning stimuli have the same effect on the response to identical c.s.n. test stimuli and on the magnitude of the after-discharge, which virtually disappears at the highest intensities of conditioning stimulation.

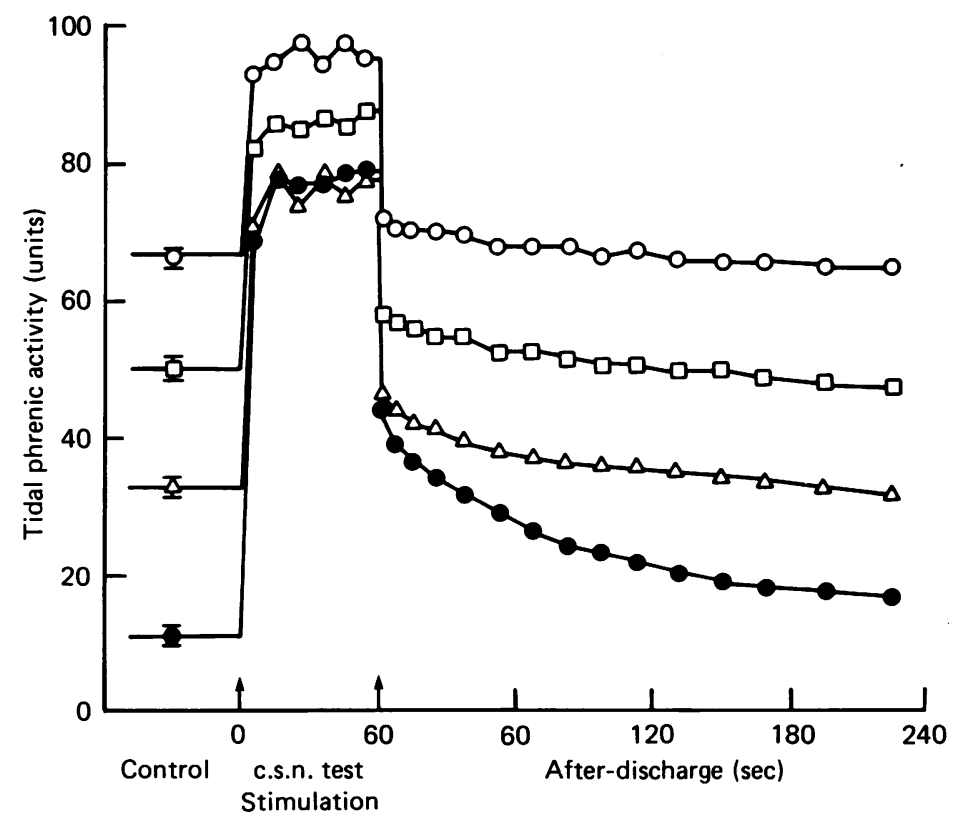


depicts the results of 66 test stimulations where the conditioning stimulus was one c.s.n. and the test stimulus the opposite c.s.n. Only the tidal phrenic activity will be graphed because the findings with minute phrenic activity were not significantly different. Both showed the same findings; that identical c.s.n. test stimuli yielded progressively smaller incremental peak responses (the difference between initial

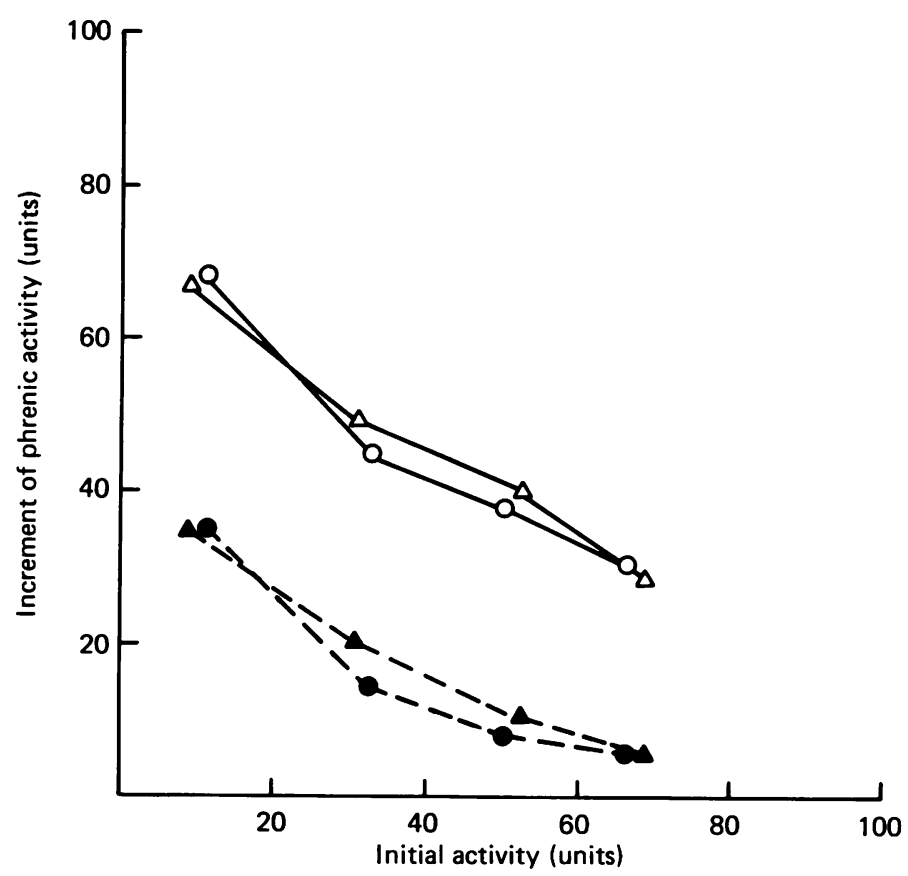

Fig. 7. Effect of the level of control, or initial, phrenic activity conditioned by continuous stimulation of the opposite c.s.n. (circles) or central chemoreceptors by hypercapnia (triangles) on the peak phrenic response $(O \Delta)$ to a c.s.n. test stimulation and on the magnitude of the respiratory after-discharge (OA) following offset of the test stimulus.

activity and peak activity) and incremental after-discharge responses (the difference between initial activity and that of the first breath after offset of stimulation) as the initial level of activity increased due to a conditioning stimulus from the opposite c.s.n. These changes are plotted in Fig. 7. This Figure shows that the magnitude of the after-discharge is also influenced by the increased level of initial activity.

Fig. 6. Responses of tidal phrenic activity in all cats to carotid sinus nerve (c.s.n.) test stimulations under isocapnic conditions but with different levels of conditioning stimulation from opposite c.s.n. The data were separated into four groups depending upon the initial (control) level of activity in each experimental run. As control activity increases, the response to a constant test stimulus of the c.s.n. progressively decreases. The magnitude of the after-discharge decreases in a similar manner. Brackets on control values are \pm S.E. Standard errors for all other points are of similar magnitude to those of the controls. 


\section{Effect of conditioning stimulation by raised $\mathrm{CO}_{2}$}

When the initial phrenic activity was increased by raising $P_{\mathrm{ET}, \mathrm{CO}_{2}}$, the increment in activity during a c.s.n. test stimulus decreased in a manner similar to that when the opposite c.s.n. was the conditioning stimulus. Fig. 5 shows an example where $P_{\mathrm{ET}, \mathrm{CO}_{2}}$ was raised from 26 torr (Fig. 5D) to 34 torr (Fig. $5 E$ ) and then to 47 torr $($ Fig. $5 F)$. The Figure shows that identical stimulations of the right c.s.n. led to

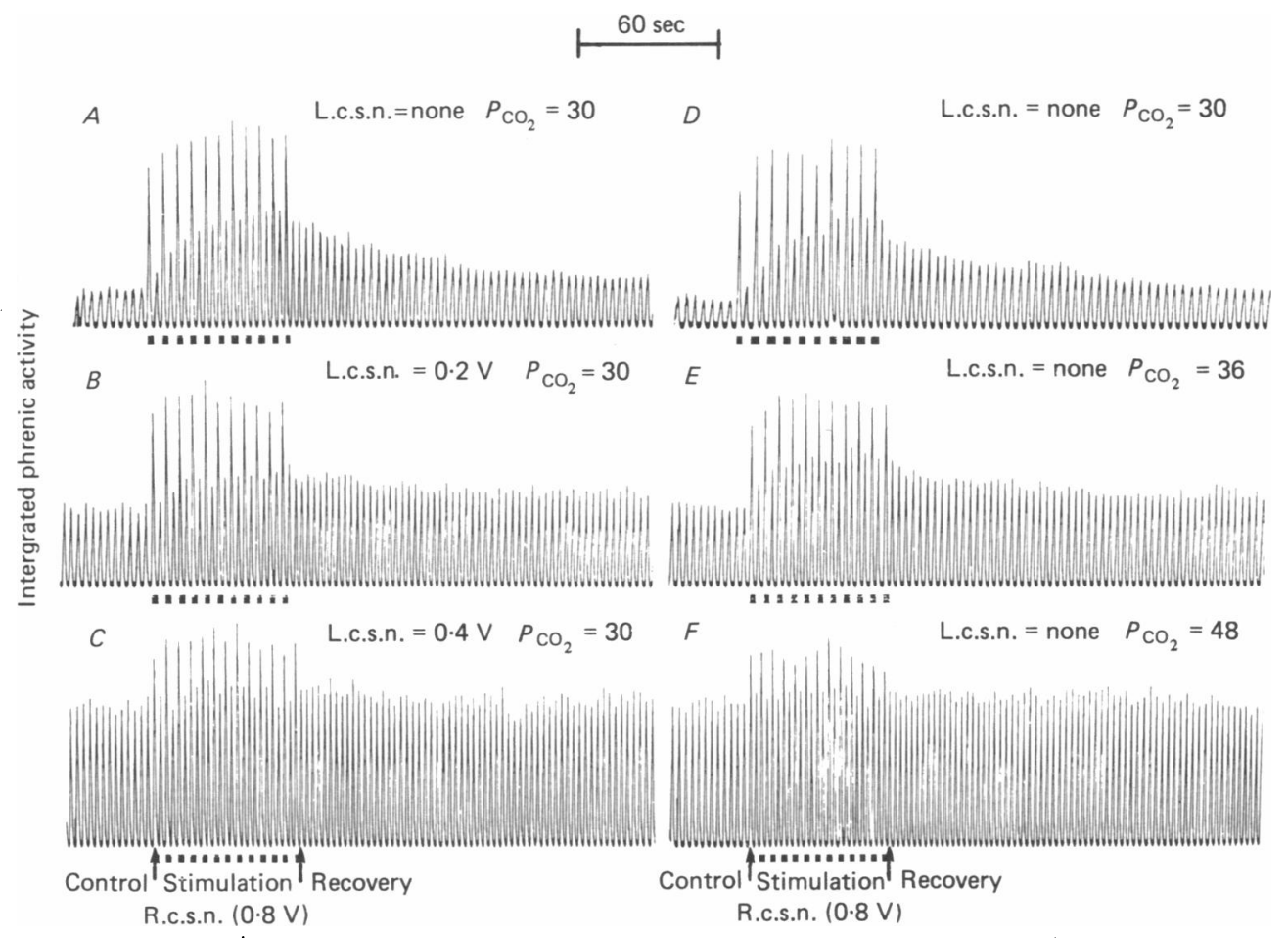

Fig. 8. Responses of phrenic activity to alternate breath carotid sinus nerve (c.s.n.) test stimulations. $A-C$, under isocapnic conditions with different intensities of conditioning stimulation from opposite c.s.n. $D-F$, with increasing hypercapnic conditioning stimulation from the central chemoreceptors.

Both types of conditioning stimuli have the same effect on the response to identical c.s.n. test stimuli and on the magnitude of the after-discharge (recovery) which virtually disappears at the highest intensities of conditioning stimulation. Note also that the direct responses to the c.s.n. test stimulus decrease as activities of unstimulated breaths increase.

progressively smaller increments over initial activity of both peak and afterdischarge activity, the latter disappearing at the highest $P_{\mathrm{ET}, \mathrm{CO}_{2}}$ (Fig. $5 F$ ).

Group data from forty-six stimulations, again divided into four groups according to the initial activity level, yielded plots which were essentially the same as when the c.s.n. provided the conditioning stimulus; as initial activity increased due to rising $P_{\mathrm{CO}_{2}}$, the same c.s.n. test stimulus yielded progressively smaller increments over initial activity of both peak activity and that due to the after-discharge mechanism. These changes are also shown in Fig. 7. 


\section{Alternate breath c.s.n. stimulation}

In some experiments, the test stimulus consisted of alternate breath instead of continuous c.s.n. stimulation. Such stimulation has been shown to yield approximately the same peak inspiratory effect as continuous stimulation but allows visualization of the slower development of the after-discharge process (see Fig. $2 B$ ). Such an experiment is exemplified in Fig. 8. In the left panels (Fig. $8 A, B$ and $C$ ) there is a progressive increase in the conditioning stimulus effected by increasing voltage on the left c.s.n. at the same end-tidal $P_{\mathrm{CO}_{2}}$. The same right c.s.n. test stimulus produces progressively smaller increments of peak phrenic activity over initial activity and of the magnitude of the after-discharge. When $\mathrm{CO}_{2}$ is the conditioning stimulus (Fig. $8 D, E$ and $F$ ) the findings are the same. At the highest level of conditioning $P_{\mathrm{CO}_{2}}$, the decaying after-discharge is absent.

The findings with alternate breath c.s.n. stimulation were the same as with continuous c.s.n. stimulation. However, the ability to visualize the rising afterdischarge activity in the alternate breath experiments allowed us to separate the increment in phrenic activity due to the direct effect of a c.s.n. stimulus from that related to the after-discharge mechanism. In this analysis the rising after-discharge is treated as another conditioning stimulus and the level of activity of the unstimulated breath following a stimulated breath, i.e. the sum of initial activity and the increment due to the after-discharge, is considered to be the conditioning level for stimulation of the next breath. Fig. 8 ( $A$ and $D$ ) shows examples of the findings, which were similar in all animals; as the conditioned level of phrenic activity rises due to the after-discharge mechanism, the increment of activity due to the direct effects of a c.s.n. test stimulation falls. Thus, the effect of conditioning by the after-discharge is similar to that of the opposite c.s.n. or $\mathrm{CO}_{2}$ stimulation.

\section{DISCUSSION}

In this study, we have determined the inspiratory output response, as measured by phrenic nerve activity, to central and peripheral chemoreceptor afferent inputs given alone and in combinations and to input from the central neural mechanism which produces the respiratory after-discharge. The findings indicate that these inputs to the respiratory controller do not add their effects algebraically; the response to combined inputs from any of the sources studied is always less than that predicted from a simple addition of the responses to the individual stimuli given alone.

In the case of the $P_{\mathrm{CO}_{2}}-\mathrm{H}^{+}$ion stimulus from the central chemoreceptors, the non-linear summation of effects of equal increments of $P_{\mathrm{CO}_{2}}$ is apparent from the curvilinear response seen in Fig. 1. This type of curve suggests that some part of the neural system subserving the response is becoming progressively saturated as input increases. It is not due to characteristics of the central chemoreceptors or direct inhibition of brainstem or spinal motoneurones by $\mathrm{CO}_{2}$ (Gill \& Kuno, 1963) because the same hypoadditive interaction occurs with opposite c.s.n. inputs, i.e., the response to two inputs is only about $\mathbf{7 0} \%$ as great as that predicted from the sum of the two individual inputs. Since the same findings obtained at all intensities of c.s.n. stimulation (Fig. 4) this result must be due not to complete saturation of some neural component but rather to a progressive saturation. 
For several years this laboratory has been reporting studies demonstrating the existence of a central neural mechanism which has the property of maintaining, for some minutes after cessation of a primary stimulus, an increased but slowly declining respiratory output (after-discharge) (Eldridge, 1974). The mechanism has been shown to be activated slowly as well (Eldridge \& Gill-Kumar, 1979, 1980a). It is activated by, but is independent of, both central and peripheral chemoreceptor inputs as well as other peripheral inputs (Eldridge, 1974) and is not dependent upon feedback from medullary output neurones (Eldridge \& Gill-Kumar, 1978). Thus it can be considered to behave as an independent neural drive to respiration whenever it is activated (Eldridge, 1980). The findings of the present study show that when the after-discharge is viewed as a conditioning stimulus, it has the same diminishing effect on the phrenic response to a c.s.n. test stimulus as when the opposite c.s.n. or $\mathrm{CO}_{2}$ are the conditioning stimuli.

Thus, with all three inputs, the carotid and central chemoreceptor afferents and the after-discharge mechanism, the result is the same; the magnitude of the response to a constant test stimulation is inversely related to the pre-existing level of activity regardless of the type of input that produced it. Such a relationship has also been reported by Kiwull, Kiwull-Schöne \& Klatt (1976).

We have considered various factors which might affect the intepretation of these results. Our preparation ruled out influences from vagal and carotid body receptors, changing input from intercostal muscle afferents and the complicating effects of hypoxia acting on brainstem neurones or on the central circulation. It is true that c.s.n. stimulation introduced an admixture of input from baroreceptor fibres along with chemoreceptor input. However, the c.s.n. test stimulus in most experiments remained constant throughout all changes in conditioning stimulation, so the baroreceptor component would have remained constant as well. Furthermore, the relative amount of baroreceptor input must have varied from cat to cat because in some arterial pressure rose or did not change and in others it fell slightly; yet this did not affect the consistency of the results. Thus, it is not likely that this factor is important.

As noted above, the findings are consistent with progressive saturation of some component of the neural pathway between afferent stimulus input and phrenic output. Single neurones (at least motoneurones) seem to have ranges in which their output is linear with increasing input (Granit, 1970), although most probably do reach a point of saturation, i.e. increasing input no longer causes an increase in output. This would not explain our findings, where saturation is progressive throughout the range of inputs and outputs. Individual neurones in a network, however, might be expected to have different thresholds and thus to saturate individually at different levels of input. Such a network would show progressive saturation of its over-all output as found here, eventually reaching a level where additional input would have no further effect. Findings similar to ours, but using ventilation as the respiratory output variable in spontaneously breathing cats with sectioned carotid sinus nerves, have been reported by Kiwull et al. (1976). They proposed that the mechanism was one of neural occlusion. However, since the effects of progressively rising $P_{\mathrm{CO}_{2}}$ only are substantially the same as with inputs from various sources, the mechanism of occlusion is not as satisfactory an explanation as that of saturation. 
Since all modes of input used in this study led to similar findings, we conclude that the neuronal structures in which saturation occurs are separate from the chemoreceptors and the after-discharge mechanism, and that they are part of the common pathway after convergence of these inputs. Because augmented breaths (sighs), gasping inspirations and calf muscle stimulation all led to higher peak levels of phrenic activity than the maxima found in the study (unpublished observations), we believe that complete saturation of phrenic motoneurones and inspiratory output neurones in the medulla had not developed. We therefore suggest that the process of progressive saturation occurs in some interneuronal pool after convergence of $\mathrm{CO}_{2}$, c.s.n. and after-discharge inputs but ahead of medullary output neurones.

Other workers have reported a curvilinear response, like ours, of peak amplitude of the phrenic neurogram (Kobayasi \& Murata, 1979) and occluded inspiratory muscle pressure (Grunstein, Younes \& Milic-Emili, 1973) to increasing $P_{\mathrm{A}, \mathrm{CO}_{2}}$. Although our findings deal only with inspiratory processes, similar curvilinear responses of tidal volume to hypercapnia have also been shown (Florez \& Borison, 1967; Grunstein et al. 1973; Kiwull, et al. 1976). Thus, even when the expiratory component of tidal volume is included, the evidence still supports the existence of a progressively saturating mechanism.

In our cats the results with minute activity were similar to tidal activity (see Figs. 1 and 4), due to small and similar effects on respiratory frequency of $\mathrm{CO}_{2}$ and c.s.n. stimulation in these vagotomized and glomectomized cats. The same may not apply to stimulation of muscle afferents because of their disproportionately greater central effect on frequency than on tidal activity (Eldridge \& Gill-Kumar, 1980b), or to other situations where frequency is in part determined by other than central mechanisms, for example $\mathrm{CO}_{2}$ administration to cats with intact vagi and carotid bodies (Florez \& Borison, 1967; Grunstein et al. 1973).

Although there is general agreement that hypercapnic and hypoxic stimuli interact multiplicatively at the site of peripheral chemoreceptors (Eyzaguirre \& Lewin, 1961; Kiwull et al. 1976), there is disagreement as to what kind of interaction between the two inputs occurs centrally. Some workers believe that the interaction is simply additive (Koepchen, Borchert, Frank, Klüssendorf, Kolbe \& Sommer, 1976; Cherniack, Euler, Homma \& Kao, 1979), others that it is multiplicative (Lahiri \& Delaney, 1975), while still others have presented evidence that it is a negative, or hypoadditive, one (Ou, Miller \& Tenney, 1976; Kiwull et al. 1976; Berger, Berger, Berndt \& Giese, 1978; Giese, Berndt \& Berger, 1978). Our studies performed in the absence of peripheral interactions, vagal input or changes of $P_{\mathrm{CO}_{2}}$, and without the central effects of hypoxia, support a conclusion that the purely central interaction is indeed a negative one.

Respiratory after-discharge. We pointed out above that the mechanism causing the respiratory after-discharge can be considered to be an independent neural drive to the respiratory controller. Its increase, by affecting the level of activity in the controller, leads to a progressively smaller response to a constant test stimulus in a way similar to c.s.n. and hypercapnic drives. We showed earlier (Eldridge, 1974) that the magnitude of the after-discharge is inversely related to the pre-existing level of activity; this was again seen here (see Fig. 7) and probably reflects again the progressive saturation of the common pathway after convergence of inputs. In 
addition, however, the after-discharge mechanism itself appears to saturate completely at a level of output below that at which the combined pathway develops complete saturation. This is shown in Figs. $5 F$ and $8 C$ where, at the highest levels of initial activity caused by conditioning stimulation of the central chemoreceptors $\left(\mathrm{CO}_{2}\right)$ or the c.s.n., no after-discharge occurs even though a test c.s.n. stimulus is capable of driving output to a higher level. The mean data in Fig. 7 suggest that complete saturation of the after-discharge occurs when initial activity of the system is about $80 \%$ of the maximum. These findings lend further support to the idea, developed more fully elsewhere (Eldridge \& Gill-Kumar, 1978, 1979; Eldridge, 1980) that the neurones generating the after-discharge are indeed separate from the respiratory controller itself.

Finally, and from a practical standpoint, the study suggests that some caution must be used in making quantitative interpretations of the meaning of changes of respiratory outputs, for example phrenic neurograms and inspiratory airway occlusion pressures, in terms of specific inputs. Since the output responses to identical inputs are a function of the existing level of activity, an attempt should be made to perform such studies at the same control level; if this is not possible the central neural interaction described here must be considered in the interpretation of the results.

The authors express their appreciation to Eve Gallman for her excellent technical assistance.

Thisstudy was supported by U.S. Public Health Service Research Grants HL-17689 and NS-11132.

Dr Millhorn was supported by U.S.P.H.S. Pulmonary Training Grant HL-07106.

\section{REFERENCES}

Berger, W., Berger, K., Berndt, J. \& Giese, K. (1978). Interaction of peripheral and central respiratory drives in cats. I. Effects of sodium cyanide as a peripheral chemoreceptor stimulus at different levels of CSF pH. Pflïgers Arch. 374, 205-210.

Cherniack, N. S., Edelman, N. H. \& Lahiri, S. (1970/1971). Hypoxia and hypercapnia as respiratory stimulants and depressants. Resp. Physiol. 11, 113-126.

Cherniack, N. S., Euler, C. von, Homma, I. \& KaO, F. F. (1979). Graded changes in central chemoreceptor input by local temperature changes on the ventral surface of the medulla. $J$. Physiol. 287, 191-211.

ELDRIDGE, F. L. (1974). Central neural respiratory stimulatory effect of active respiration. J. appl. Physiol. 37, 723-735.

EldRIDGe, F. L. (1975). Relationship between respiratory nerve and muscle activity and muscle force output. J. appl. Physiol. 39, 567-574.

EldRIDGE, F. L. (1980). Subthreshold respiratory activity and after-discharge. Resp. Physiol. 39, 327-343.

EldRIDGe, F. L. \& Gill-KumaR, P. (1978). Lack of effect of vagal afferent input on central neural respiratory afterdischarge. $J$. appl. Physiol. 45, 339-344.

Eldridge, F. L. \& Gill-Kumar, P. (1979). Central neural drive mechanisms and respiratory afterdischarge - the 'T-pool' concept. In Central Nervous Control Mechanisms in Breathing, ed. Euler, C. von \& Lagercrantz, H., pp. 101-113. Oxford: Pergamon.

Eldridge, F. L. \& Gill-Kumar, P. (1980a). Central neural respiratory drive and afterdischarge. Resp. Physiol. 40, 49-63.

Eldridae, F. L. \& Gill-Kumar, P. (1980b). Central respiratory effects of $\mathrm{CO}_{2}$, and carotid sinus nerve and muscle afferents. J. Physiol. 300, 75-87.

EyzaquirRe, C. \& Lewin, J. (1961). Chemoreceptor activity of the carotid body of the cat. $J$. Physiol. 159, 222-237.

Florez, J. \& Borison, H. L. (1967). Tidal volume in $\mathrm{CO}_{2}$ regulation: peripheral denervations and ablation of area postrema. Am. J. Physiol. 212, 985-991. 
GIESE, K., BERndT, J. \& BERGER, W. (1978). Interaction of central and peripheral respiratory drives in cats. II. Peripheral and central interaction of hypoxia and hypercapnia. Pflügers Arch. 374, 211-217.

Gill, P. K. \& Kuno, M. (1963). Properties of phrenic motoneurones. J. Physiol. 168, $258-273$.

Granit, R. (1970). The Basis of Motor Control, pp. 135-141. London: Academic.

Grunstein, M. M., Younes, M. \& Milic-EmiLI, J. (1973). Control of tidal volume and respiratory frequency in anaesthetized cats. $J$. appl. Physiol. 35, 463-476.

Kiwull, P., Kiwull-Schöne, H. \& Klatt, W. (1976). Interaction of central and peripheral respiratory drives: differentiation between the role of stimuli and afferents. In Acid-Base Homeostasis of the Brain Extracellular Fluid and the Respiratory Control System, ed. LoEscHCKE, H. H., pp. 146-156. Stuttgart: Thieme.

Kobayasi, S. \& Murata, K. (1979). Phrenic activity during severe hypercapnia in vagotomized rabbits. J. appl. Physiol. 47, 91-97.

Kofpchen, H. P., Borchert, J., Frank, Ch., Klüssendorf, D., Kolbe, K. \& Sommer, D. (1976). Respiratory center input-output relations in response to different afferences. In Respiratory Centres and Afferent Systems, ed. Duron, B., vol. 59, pp. 73-84. Paris: INSERM.

Lahiri, S. \& Delaney, R. G. (1975). Relationship between carotid chemoreceptor activity and ventilation in the cat. Resp. Physiol. 24, 267-286.

Mustafa, M. E. K. Y. \& Purves, M. J. (1972). The effect of $\mathrm{CO}_{2}$ upon discharge from slowly adapting stretch receptors in the lungs of rabbits. Resp. Physiol. 16, 197-212.

Ou, L. C., Miller, M. J. \& Tenney, S. M. (1976). Hypoxia and carbon dioxide as separate and interactive depressants of ventilation. Resp. Physiol. 28, 347-358.

Smith, D. M., Mercer, R. R. \& Eldridge, F. L. (1978). Servo-control of end-tidal $\mathrm{CO}_{2}$ in paralyzed animals. J. appl. Physiol. 45, 133-136.

Sorenson, S. C. (1970). Ventilatory acclimatization to hypoxia in rabbits after denervation of peripheral chemoreceptors. J. appl. Physiol. 29, 81-92.

TEnney, S. M. \& OU, L. C. (1977). Ventilatory response of decorticate and decerebrate cats to hypoxia and $\mathrm{CO}_{2}$. Resp. Physiol. 29, 81-92. 